\title{
AUTONOMOUS SYSTEM IDENTIFICATION AND CONTROL OF MACE II USING THE FREQUENCY DOMAIN EXPERT ALGORITHM
}

\author{
Larry D. Davis ${ }^{1}$, James A. King ${ }^{1}$, Scott W. Greeley ${ }^{1}$, and Dr. David C. Hyland ${ }^{2}$ \\ ${ }^{1}$ Planning Systems, Inc., Melbourne, FL, www.psi-controls.com \\ ${ }^{2}$ Chairman, Department of Aerospace Engineer, University of Michigan, Ann Arbor, MI
}

\begin{abstract}
$\underline{\text { Abstract }}$
The Frequency Domain Expert (FDE) adaptive identification and control design algorithm will be applied to the Middeck Active Control Experiment (MACE) during a re-flight of the experiment. FDE was recently developed by the authors to address the need for an algorithm that requires only local measurement, has predictable convergence properties, and yields performance in terms easily understood by controls system engineers. Ground testing to date has produced FDE controllers that reduce line-of-sight error by more than $22 \mathrm{~dB}$, only a few $\mathrm{dB}$ less than the best performance attained via offline; fixed-gain LQG controllers during the original MACE flight.
\end{abstract}

\section{Introduction}

The last ten years have witnessed an explosion in the study of adaptive control methods because of their potential to greatly reduce the required labor in design and modeling efforts, recover autonomously from failures, reduce the need for engineering support in operation, and increase performance by reducing design margins. Application of this technology to the control of precision structures, however, has been hampered by three major difficulties: the lack of reliable methods for predicting the convergence and performance of the algorithms; the prohibitive computing resources needed to implement the technology; and potential users' lack of familiarity with adaptive methods.

MACE II, a SBIR Phase II program awarded to Planning Systems, Inc. and sponsored by the Air Force Research Lab (AFRL), focuses directly on resolving these problems. The program works to overcome potential users' lack of familiarity with adaptive control methods by demonstrating the power of adaptive control on a space flight experiment using the Frequency Domain Expert algorithm. This demonstration is based on the re-flight of the Middeck Active Control Experiment (MACE) (shown in Figure 1), a highly successful testbed first flown on a Shuttle mission in 1995. The MACE II program is a team effort of PSI, Payload Systems Inc., the Univ. of Michigan, and AFRL.

The Frequency Domain Expert (FDE) is a recent invention by Dr. David Hyland at the University of Michigan and members of PSI's Melbourne Controls Group. Its development was motivated by the need for an algorithm for feedback which requires only a local measurement, has predictable convergence properties, and yields performance in terms easily understood by controls system engineers. The Frequency Domain Expert algorithm can be thought of as a nonlinear mapper that measures and inspects the frequency response characteristics of the system in order to arrive at a control design that meets frequency domain performance specifications. No adaptation of an internal system model is needed. Instead, the algorithm takes frequency response measurements of the system, applies a nonlinear mapping to arrive at a stabilizing control law, closes the loop, and if needed takes a new measurement to begin the process again. Once it has converged to a controller which meets the performance specifications, it no longer performs frequency response measurements but switches to a passive monitoring of the measurement, waiting for a change in the signature of the signal which indicates that the system dynamics have changed. If a change occurs, the algorithm begins adapting again.

This paper describes the Mace II flight experiment hardware and controller, the identification and control synthesis portions of the FDE algorithm, and results achieved during ground testing on the MACE-II test facilities at AFRL.

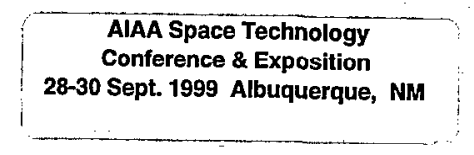

${ }^{*}$ Copyright $\odot 1999$ by the American Institute of Aeronautics and Astronautics Inc. All rights rescrved. 

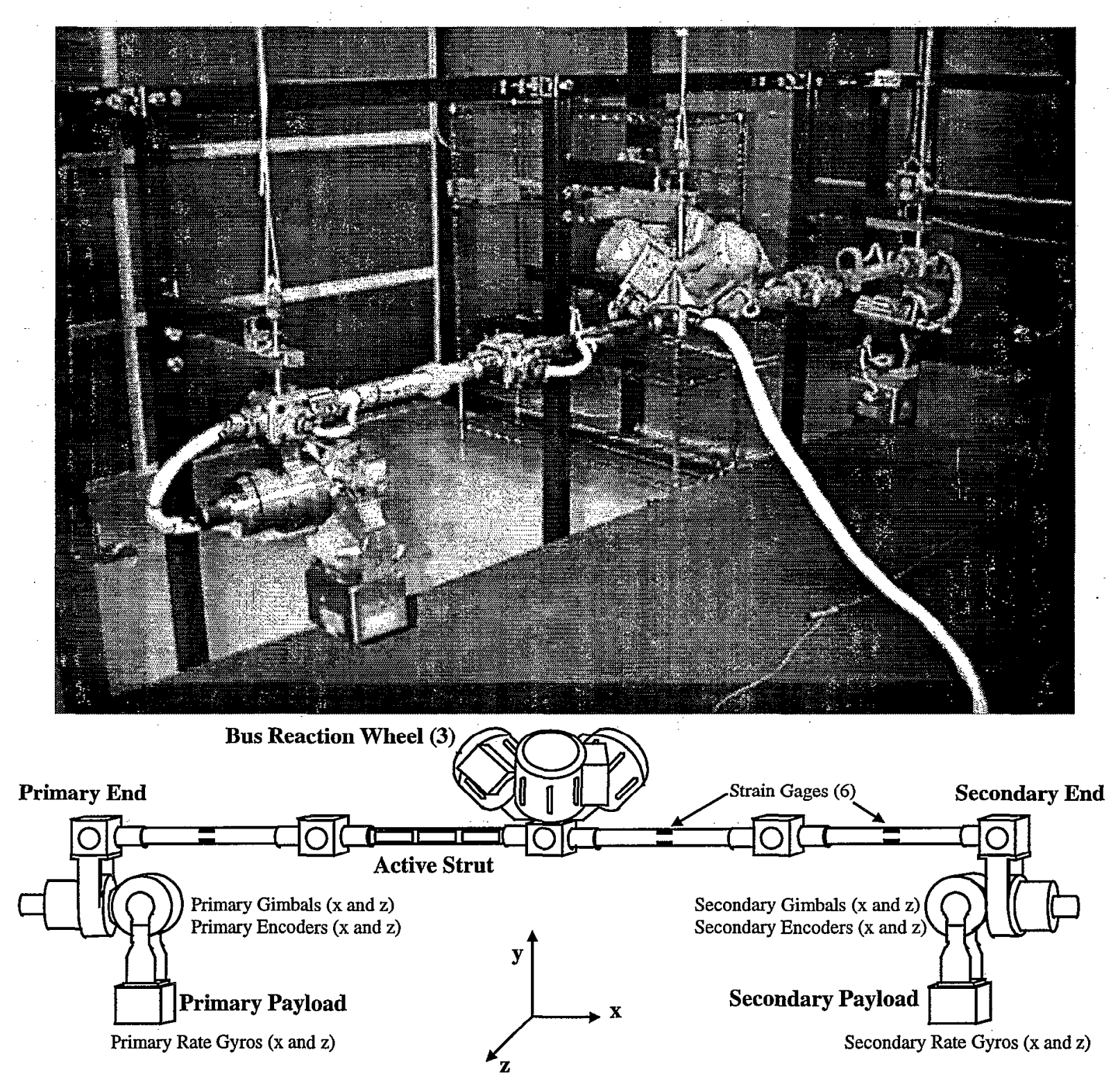

Figure 1 Photo and Sketch of Multibody Platform Test Article of MACE (photo taken at AFRL facilities)

\section{MACE II Experiment Description}

The Middeck Active Control Experiment (MACE) was originally developed for a Space Shuttle flight experiment that flew on STS-67 in 1995. ${ }^{1}$ Its primary mission was to investigate methods for achicving high precision pointing and vibration control of spacecraft and satellites. The MACE II program is a re-flight of the experiment with refurbished hardware (with minor modifications) and refined software. Its mission is to investigate adaptive control and identification algorithms.
MACE consists of the Multibody Platform (MBP) test article, the Experiment Support Module (ESM), and the $\mathrm{Ku}$-Band Interface System (KIS) used for uplink and downlink of data during flight. The MBP was designed to represent a satellite with multiple, pointing payloads with a fundamental bending frequency below $2 \mathrm{~Hz}$. The MBP, shown in Figure 1 in its Configuration 1, has a long ( $\sim 6 \mathrm{ft}$.) flexible bus comprised of four beam sections with actuators and sensors mounted at various locations. Payloads that can be independently pointed in two axes are mounted at each end of the bus. The payloads are pointed using gimbals. Each gimbal has an encoder attached to its axis for measuring its relative 
angle and closing a low bandwidth servo loop. Two rate gyros are mounted on the 'payload' side of the gimbals for measuring the payload angular rates. Attached at the mid-point of the flexible bus are three reaction wheels, and co-located rate gyros. Each wheel uses a tachometer, fed back to a local rate loop, for controlling its speed. An active strut (comprised of piezo elements) is used as one of the four sections of the flexible bus. In addition, several strain gages are mounted at locations along the flexible bus to measure its bending strain. For ground testing, the MBP is supported using a pneumatic suspension system. In spacc, it will be lightly tethered to restrain its range of motion. An umbilical cord (the white cable bundle attached near the reaction wheels) is used to connect actuator and sensor signals from the MBP to the ESM.

The ESM provides the electrical power and brains for controlling the MBP. It consists of the electronics for driving the actuators, conditioning and measuring the sensors, and software for implementing the control algorithms. Real-time data is stored on a removable hard drive for post-processing of the results. Real-time control software is executed on the Digital Signal Processing Module (DSPM) within the ESM, which is a Texas Instruments TMS320C30 $40 \mathrm{MHz}$ DSP. One significant software modification made earlier in the MACE II program was to allow investigators to implement their own control code (the original MACE flight experiment allowed implementation of only linear, time-invariant, state-space controllers). The investigator's code is composed of initialization, background, foreground, and shutdown functions. The initialization and shutdown functions are each only executed once, at the beginning and end, respectively, during the software program's execution. The foreground functions, called at a constant sample rate of $500 \mathrm{~Hz}$, are used to implement the real-time tasks such as computing the controller. The background function, executed whenever the processor has free time, is used for implementing those tasks that do not need to be executed at a fixed interval such as control design.

We have performed our ground testing with the MPB in the configuration shown in Figure 1. The two gimbals at the secondary end are used to disturb the bus and primary payload. Two independent, random disturbance signals filtered by $50 \mathrm{~Hz}$ low pass filters drive the position commands of the two gimbals. Our performance objective is to reduce the Primary Payload inertial pointing angle about the $\mathrm{x}$ and $\mathrm{z}$ axes - referred to later as line-of-sight error. To accomplish this, we are using various combinations of the primary payload gimbals and flexible bus reaction wheels as control actuators, and the primary payload rate gyros and rate gyros co-located with the reaction wheels as control sensors. It should be noted, during our testing we used the 'baseline' servo controller at all times (even during the so called 'open-loop') to maintain a low bandwidth position loop on the gimbals.

\section{Frequency Domain Expert Algorithm}

The FDE algorithm designs a high-gain feedback, single-input, single-output control loop for a specific sensor/actuator pair. A simple executive sequences the designs of the sensor/actuator loop to achieve the overall objective. The identification and control synthesis portions are scquenced as follows. At startup, a FRF measurement is taken over the control bandwidth of interest. Then, using this measurement and a specified performance objective, the first iteration of the FDE controller is designed. At this point, either the controller is implemented and the FRF (now closedloop) is measured again, or the closed-loop FRF is computed directly from the previous FRF measurement. Which method to use depends primarily on the accuracy of FRF measurement. Obviously, reidentifying the FRF at each iteration will increase the convergence time. Next, another FDE control design synthesis iteration is computed, and the above steps are repeated until the controller meets its performance objective, or converges. The identification and control synthesis algorithms arc described below.

\section{Adaptive Identification Algorithm:}

In order for the Frequency Domain Expert algorithm to calculate the parameters of a feedback control law, it first must learn the frequency response function (FRF) from the actuator command to the local feedback measurement. To this end, we devised an adaptive identification algorithm that sequentially identifies the response at each frequency point. We chose an adaptive method over FFT-based, FRF estimates because 1) it will provide more accurate FRF estimates, especially in high signal-to-noise environments, and 2) it has fewer total FRF points, but finer frequency resolution where needed. Moreover, the FDE control design does not require the FRF be identified at a constant frequency interval. The adaptive identification method has the following features:

- a sinusoidal signal is commanded to the actuator at discrete frequency points

- the measurement and excitation signals are narrowband filtered to reduce non-linear effects and minimize out-of-band noise effects

- the frequency response is identified at each frequency point using a modified adaptive least- 
mean-square (LMS) approach with guaranteed convergence to the noise floor

- frequency spacing between FRF points is adaptively selected based on the predicted change in the response - finer frequency resolution is used in regions of large change (e.g. at structural modes); back stepping is allowed if the actual change in the FRF is too large

- the actuator command can be adaptively selected to maintain a specified target measurement amplitude
Prinary $\mathrm{X}$ rate gyro. The adaptive FRF has 404 points, with many of the points concentrated near the modes (for example, 30 points are concentrated about the 1.6 $\mathrm{Hz}$ mode as highlighted in the expanded view $-0.02 \mathrm{~Hz}$ was set as the finest frequency spacing). The time required for the adaptive $\mathrm{ID}$ was appr. 5 minutes. A comparable FFT-based H1 estimate (4096 points, 30 averages) would have a fixed frequency spacing of 0.12 Hz. Figure 3 shows the adaptive ID FRF measured for Primary $\mathrm{Z}$ gimbal to Primary $\mathrm{Z}$ rate gyro.

Figure 2 shows a FRF measured using the adaptive identification method for the Primary $X$ gimbal to

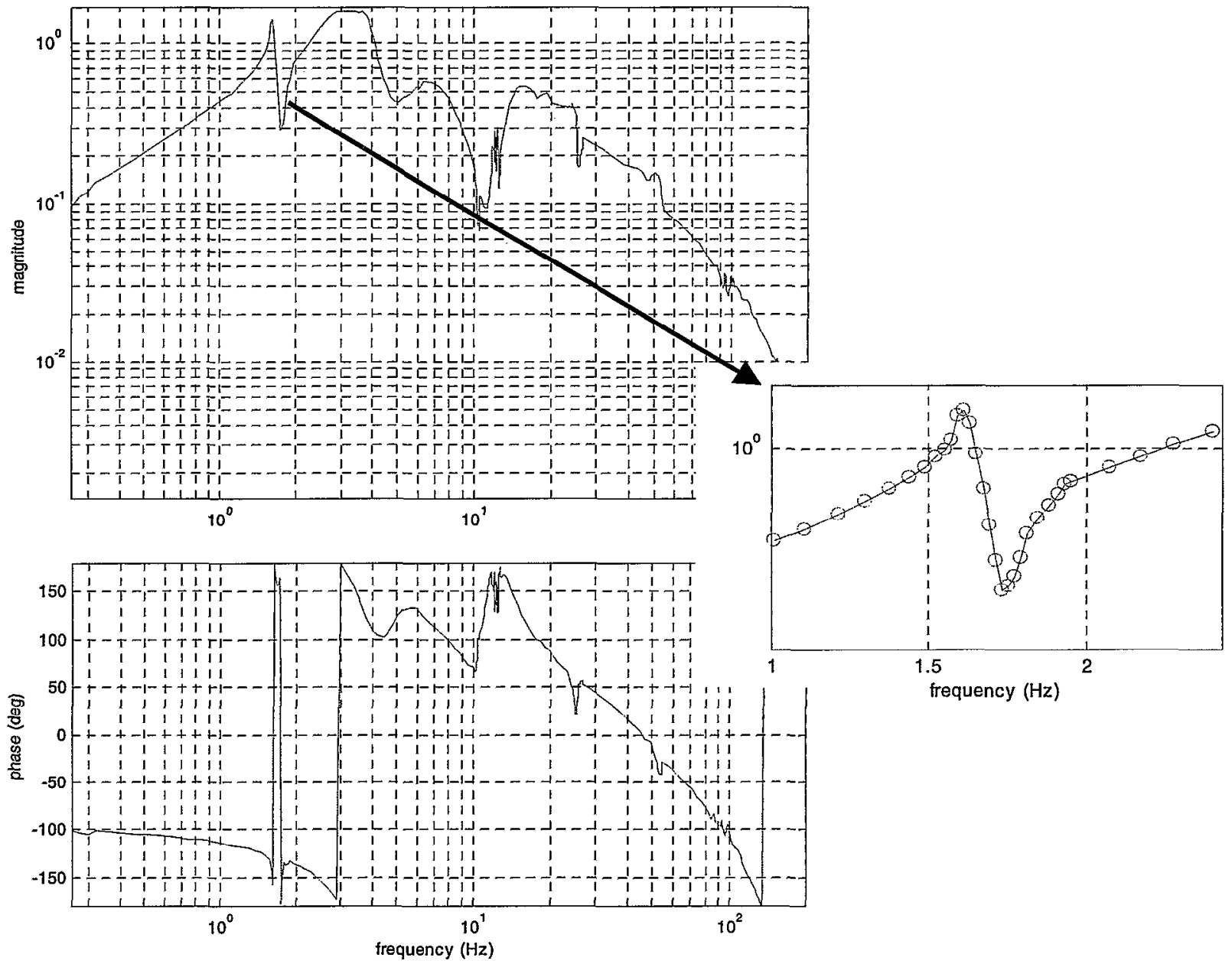

Figure 2 Primary X Gimbal to Primary X Rate Gyro FRF Measured by Adaptive ID 
(c)1999 American Institute of Aeronautics \& Astronautics or published with permission of author(s) and/or author(s)' sponsoring organization.
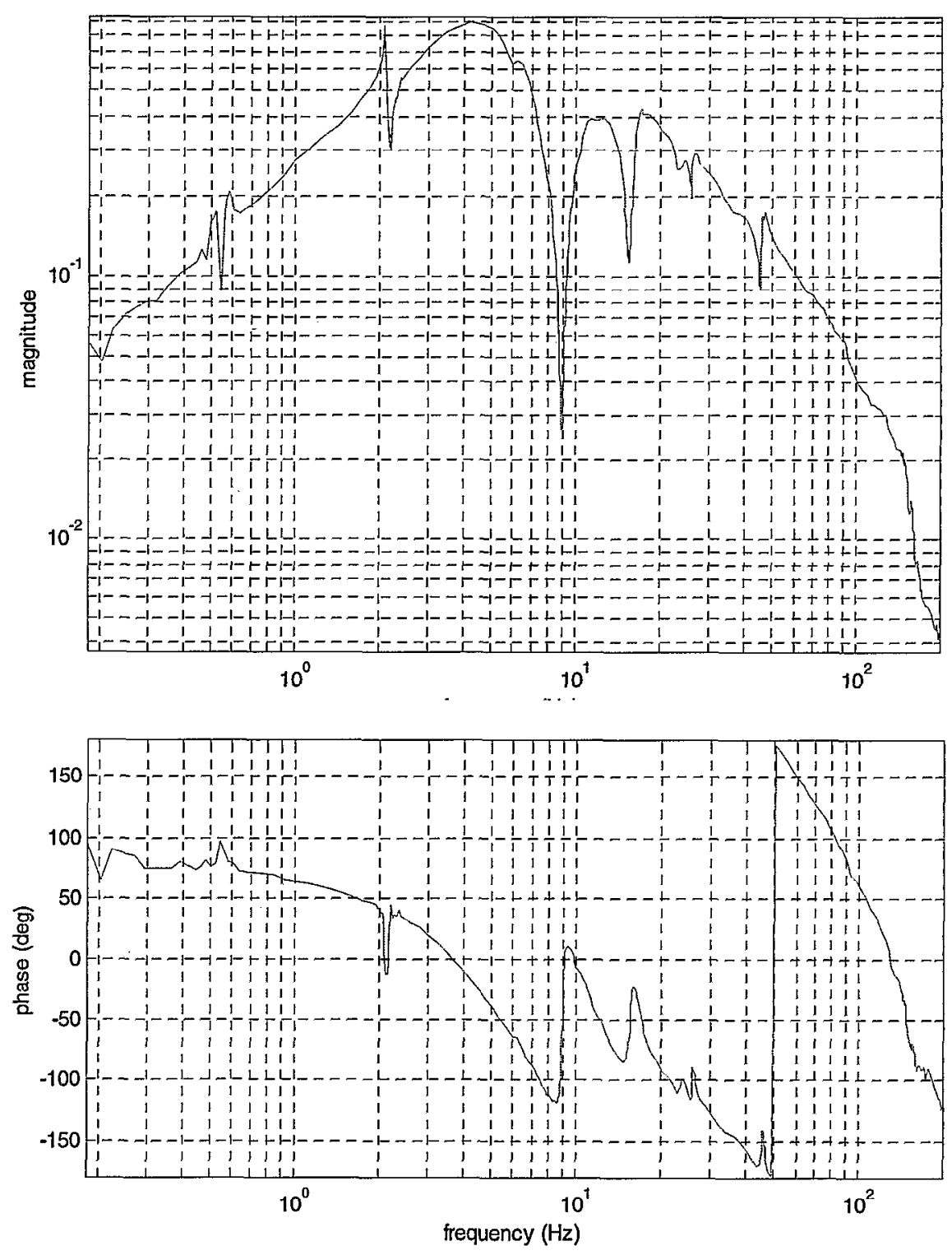

Figure 3 Primary Z Gimbal to Primary Z Rate Gyro FRF Measured by Adaptive ID

\section{Control Design Synthesis:}

The FDE control design algorithm uses measured FRF data directly, rather than using or constructing a model of the system. It constructs a control law in the frequency domain by sequentially designing low-order filters to attenuate performance violations and to stabilize cross-over points. A performance objective is specified as a frequency-domain performance bound. For structural vibrations problems, such as the MACE II programs, the performance bound will usually be specified in one of two ways: 1) an attenuation factor times the measured FRF, or 2) an attenuation factor times the measured open-loop PSD. The first method is useful for implementing co-located active damping control laws, while the second is better suited for directly attenuating a performance measure (such as line-of-sight error) due to a disturbance (such as secondary disturbance torques): Gain and phase margins, the standard parameters from classical control design, are specified by the user to enforce stability margins when designing the filters.

American Institute of Aeronautics and Astronautics 
The FDE control design process is performed in several steps as follows.

1. The frequency response function from actuator to sensor $\boldsymbol{G}(\omega)$ is determined. Upon the first iteration of the sequence no controller has been defined, therefore $\boldsymbol{G}(\omega)$ is the open loop FRF. After the first time through, then $G(\omega)$ can either be computed from the open-loop FRF and controller, or identified from scratch using the adaptive ID method.

2. A pre-phase compensator is used during first iteration to smooth out sharp transitions in FRF phase within the roll-off region of the controller.

3. The frequency point of worst violation of the performance bound is determined. Using the magnitude of the violation and the local phase of $G(\omega)$, the algorithm initializes the controller $K(\omega)$ by designing a bandpass filter which will reduce the magnitude of the performance requirement over the largest possible frequency band.

4. Notch filters are used, as required, to gain stabilize the loop transfer function $G(\omega) K(\omega)$, and $K(\omega)$ is augmented with the notches.

5. The new controller $K(\omega)$ is combined in parallel with the previous controller (from previous iterations), and the closed-loop FRF and performance requirement are updated.

6. This process is repeated using the closed-loop FRF and performance requirement until the controller is deemed converged. Several conditions can be used to signify convergence of the algorithm, including the closed loop response meeting the performance objective; a maximum number of controller states being used; or $K(\omega)$ not changing more than a specified tolerance.

\section{MACE I Ground Test Results}

Ground tests have been performed on the MACE II hardware at the AFRL facilities to check-out and validate our algorithms, and their coded implementation, at various points during their development. We will be reporting only the results from our last visit, because this represents our most mature algorithms. We used the FDE adaptive identification and control design algorithm to identify and design controllers to reduce the rms line-of-sight (LOS) error of the Primary Payload for three cases: 1) Primary X only, 2) Primary Z only, and 3) Primary X and $Z$. The LOS error was estimated by integrating the rate gyro measurement. We use the nomenclature BRG $\equiv$ Bus Rate Gyro, PRG $\equiv$ Primary (Payload) Rate Gyro, $\mathrm{BRW} \equiv$ Bus Reaction Wheel, PG $\equiv$ Primary (Payload) Gimbal.

Case 1: Our objective of the first case was to attenuate LOS error for Primary $X$. The following shows the identification and control design sequence used:

- identify BRGx/BRWx FRF, compute and implement its FDE controller using FRF based performance requirement

- identify PRGx/PGx FRF, compute and implement its FDE controller using PSD based performance requirement ( 2 controllers implemented)

Figure 4 shows the rms LOS error indicating $23.9 \mathrm{~dB}$ attenuation of LOS error with both controllers closed, and $6.3 \mathrm{~dB}$ attenuation with just the Bus X closed. The amplification near a $50 \mathrm{~Hz}$ cross-over point was due to the residual inaccuracy in the $I D$ process. This amplification could be reduced by performing another ID with the controllers on, and re-applying the FDE control design process to the 'closed-loop' FRF.

Case 2: Our objective of the second case was to attenuate LOS error for Primary $Z$. The following shows the identification and control design sequence used:

- identify BRGz/BRWz FRF, compute and implement its FDE controller using FRF based performance requirement

- identify PRGz/PGz FRF, compute and implement its FDE controller using PSD based performance requirement ( 2 controllers implemented)

Figure 5 shows the rms LOS error indicating $15.4 \mathrm{~dB}$ attenuation of LOS error with both controllers closed, and $0.1 \mathrm{~dB}$ attenuation with just the Bus $\mathrm{Z}$ closed.

Case 3: Our objective of the third case was to attenuate LOS error for both Primary $\mathrm{X}$ and $\mathrm{Z}$ axes. The following shows the identification and control design sequence used:

- identify BRGx/BRWx FRF, compute and implement its FDE controller using FRF based performance requirement

- identify BRGz/BRWz FRF, compute and implement its FDE controller using FRF based performance requirement ( 2 controllers implemented)

- identify PRGx/PGx FRF, compute and implement its FDE controller using PSD based performance requirement ( 3 controllers implemented) 
- identify PRGz/PGz FRF, compute and implement its FDE controller using PSD based performance requirement (all 4 controllers implemented)

Figure 6 shows the rms LOS error indicating $22.4 \mathrm{~dB}$ attenuation of LOS error with all four controllers closed, and $5.5 \mathrm{~dB}$ attenuation with just the two Bus controllers ( $\mathrm{x}$ and $\mathrm{z}$ ) closed. This compares with 20 to $27 \mathrm{~dB}$ performance attenuation achieved using various fixed-gain, LQG controllers during the original MACE flight experiment. Our four FDE controllers had a total of 66 states. The amplification near a $50 \mathrm{~Hz}$ cross-over point was due to the residual inaccuracy in the ID process. This amplification could be reduced by performing another ID with the controllers on, and reapplying the FDE control design process to the 'closedloop' FRF.

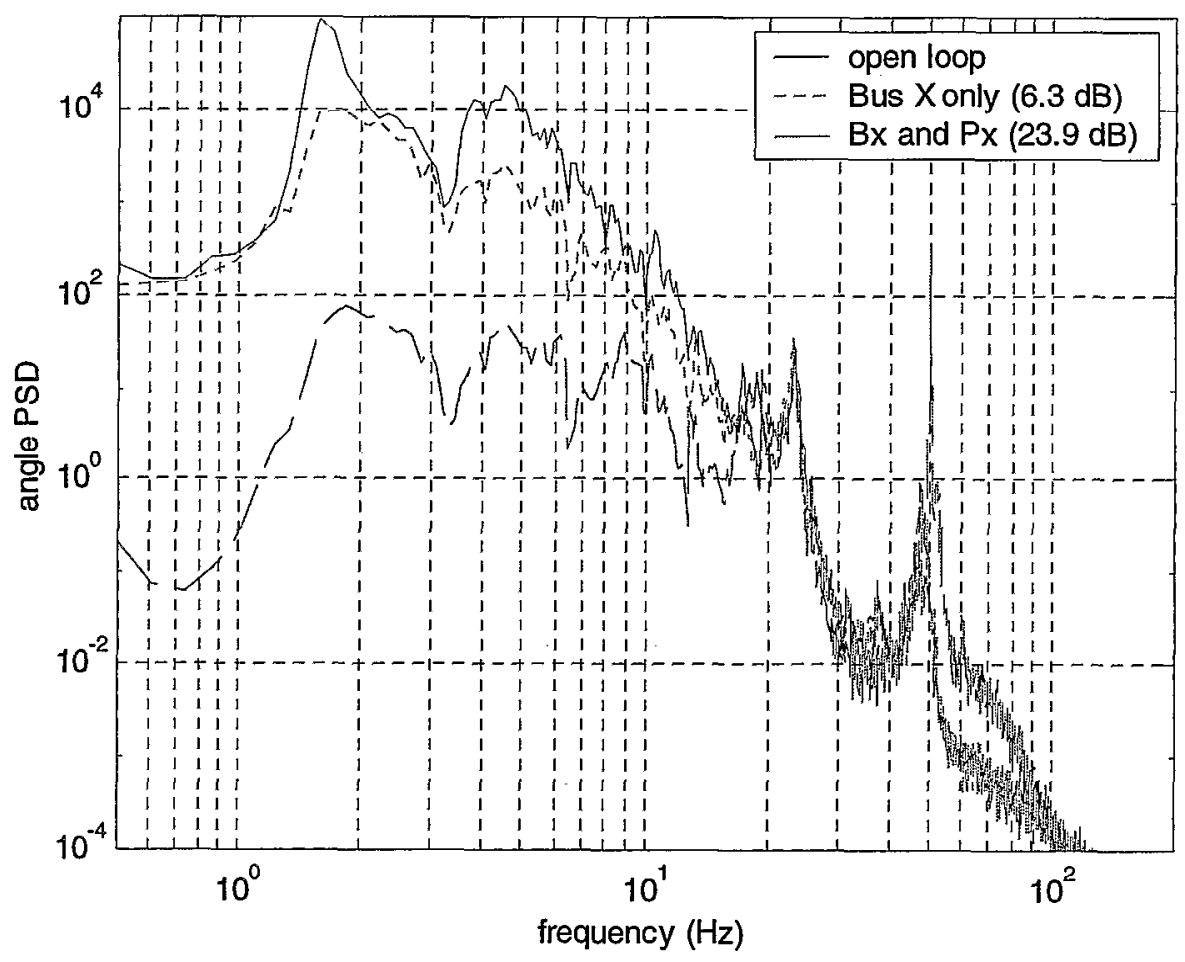

Figure 4 Primary X LOS Error - Open Loop, with Bus Loop Closed, with all Loops Closed 


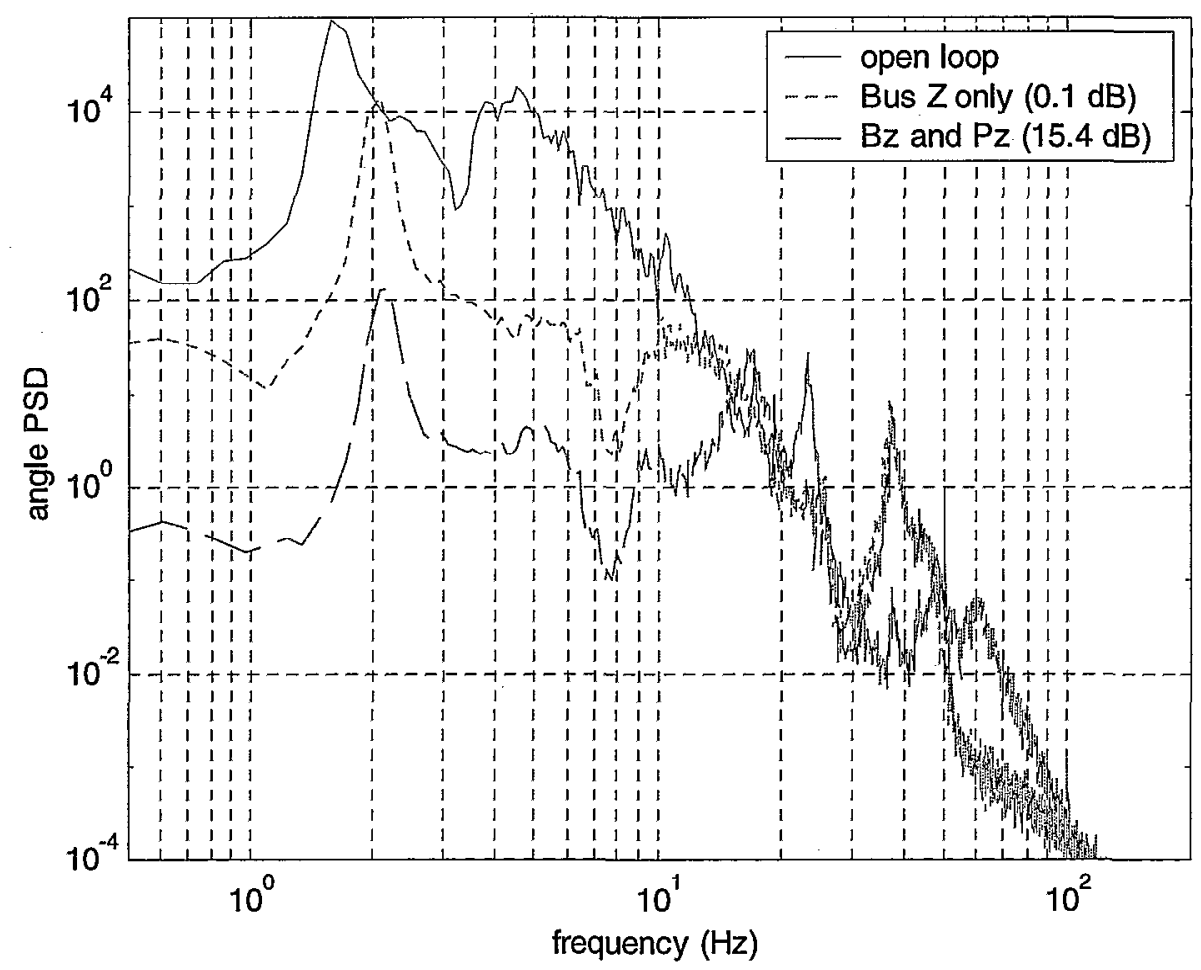

Figure 5 Primary Z LOS Error - Open Loop, with Bus Loop Closed, with all Loops Closed

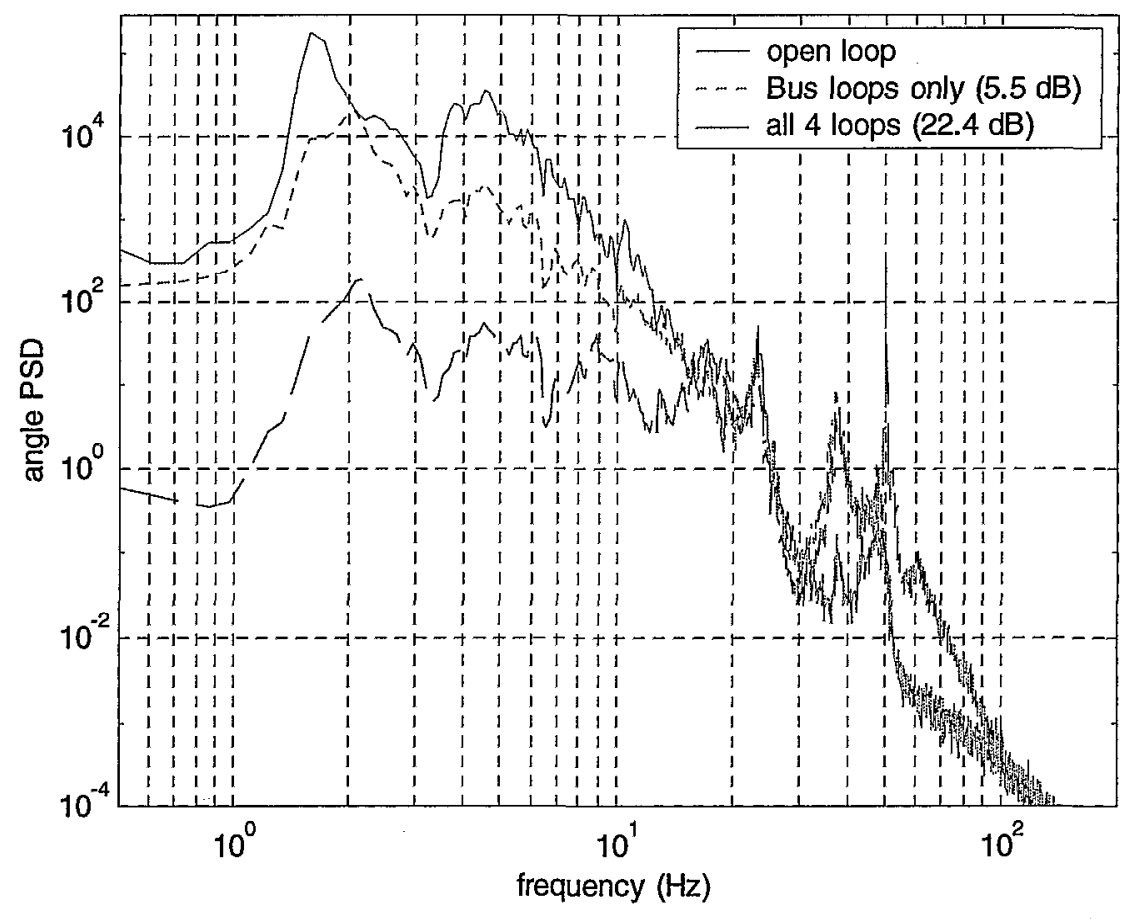

Figure 6 RMS LOS Error - Open Loop, with Bus Loops Closed, with all Loops Closed

Amcrican Institutc of Aeronautics and Astronautics 


\section{Conclusions}

Ground tests on the MACE II testbed have shown very encouraging results for the FDE algorithm. We have seen some of the benefits of FDE:

- it is an entirely on-line adaptive method, with no apriori modeling required

- the gain/phase margins are enforced at each iteration

- its controller is not plant model-order limited, instead it is limited only by the available FRF points

- we achieved $22 \mathrm{~dB}$ of line-of-sight error attenuation using only decentralized loops, which is comparable with centralized controller results obtained during the original MACE flight experiment

\section{Acknowledgements}

The authors wish to thank Mr. Rory Ninneman at the Air Force Research Lab in Albuquerque, NM for his assistance during this project.

\section{References}

1. Miller, D.W., Crawley, E.F., How, J.P., Liu, K., Campbell, M.E., Grocott, S.C.O., Glacsc, R.M. and Tuttle,T.D., "The Middeck Active Control Experiment (MACE): Summary Report", MIT Space Engineering Research Center Report, SERC \#7-96, JUNE 1996.

2. Denoyer, K.K., Hyland, D.C., Davis, L.D., and Miller, D.W., "MACE II: A Space Shuttle Experiment for Investigating Adaptive Control of Flexible Spacecraft", AIAA-98-4319, AIAA Guidance, Navigation, and Control Conference, Boston, MA, August 10-12, 1998. 